\title{
The Retentivity of Chaos under Topological Conjugation
}

\author{
Tianxiu Lu, ${ }^{1}$ Peiyong $\mathrm{Zhu}^{2}$ and Xinxing $\mathrm{Wu}^{2}$ \\ ${ }^{1}$ Institute of Science, Sichuan University of Science and Engineering (SUSE), Zigong 643000, China \\ ${ }^{2}$ School of Mathematical Sciences, University of Electronic Science and Technology of China (UESTC), Chengdu 611731, China
}

Correspondence should be addressed to Tianxiu Lu; lubeeltx@163.com

Received 17 October 2013; Accepted 10 December 2013

Academic Editor: Yuncai Wang

Copyright (c) 2013 Tianxiu Lu et al. This is an open access article distributed under the Creative Commons Attribution License, which permits unrestricted use, distribution, and reproduction in any medium, provided the original work is properly cited.

\begin{abstract}
The definitions of Devaney chaos (DevC), exact Devaney chaos (EDevC), mixing Devaney chaos (MDevC), and weak mixing Devaney chaos (WMDevC) are extended to topological spaces. This paper proves that these chaotic properties are all preserved under topological conjugation. Besides, an example is given to show that the Li-Yorke chaos is not preserved under topological conjugation if the domain is extended to a general metric space.
\end{abstract}

\section{Introduction and Preliminaries}

The existence of chaotic behavior in deterministic systems has attracted researchers for many years. In engineering applications such as biological engineering, imaging, encryption, and chaos control, chaoticity of a system is an important subject for investigation. The complexity of a topological dynamical system is intensively discussed since the term chaos is introduced by Li and Yorke [1] in 1975. However, the definition of chaos in the sense of Li-Yorke is inconveniently in research of engineering applications. In 1989, Devaney [2] stated another known definition of chaos called "Devaney chaos" today. A map $f$ is said to be chaotic in the sense of Devaney (or DevC for short) on a close set $I$ if $f$ is transitive on $I$, the set of periodic points of $f$ is dense in $I$, and $f$ has sensitive dependence on initial conditions.

In 1992, Banks et al. [3] proved that if $f:(X, d) \rightarrow$ $(X, d)$ is transitive and has dense periodic points, then $f$ has sensitive dependence on initial conditions, where $(X, d)$ is a metric space which has no isolated point. This causes that Devaney chaos is preserved under topological conjugation on general infinite metric spaces. If sensitivity is replaced by some other dynamical properties, such as weakly mixing (WMix), mixing (Mix), or exact, then we obtain stronger notions of chaos comparing with the original one (see [4-7]), which are called Weak Mixing Devaney chaos (WMDevC), Mixing Devaney chaos (MDevC), and Exact Devaney chaos (EDevC), respectively.
From [7], on metric spaces, implications

$$
\mathrm{EDevC} \Longrightarrow \mathrm{MDevC} \Longrightarrow \mathrm{WMDevC} \Longrightarrow \mathrm{DevC}
$$

immediately follow from their definitions. Since an invertible map may not be topologically exact, then $\mathrm{MDevC}$ does not imply EDevC. In [8], Carnnrl gave a dynamical system which is WMix but not Mix on a compact metric space. And an example in [9] is constructed to show that there exists a DevC map that is not WMDevC. Hence, the inverse of (1) is not proper.

This paper extends the definitions of DevC, WMDevC, $\mathrm{MDevC}$, and EDevC to topological spaces and proves that topological conjugation preserves these chaotic properties. Moreover, we use an example to show that Li-Yorke chaos is not preserved under topological conjugation on metric spaces.

Throughout this paper, let

$$
\mathbb{N}=\{0,1, \ldots\}, \quad \mathbb{Z}^{+}=\{1,2, \ldots\} .
$$

The open neighborhood system of a point $x \in X$ is denoted by $\mu(x)$. The set of periods and periodic points of a continuous self-map $f$ are denoted by $P(f)$ and $\operatorname{Per}(f)$, respectively.

Definition 1 (see [10]). A map $h: X \rightarrow Y$ is called a homeomorphism if it is one-to-one and onto, and both $h: X \rightarrow Y$ and $h^{-1}: Y \rightarrow X$ are continuous. 
Definition 2 (see [10]). Let $X$ and $Y$ be two topological spaces and let $f: X \rightarrow X$ and $g: Y \rightarrow Y$ be two continuous maps. Maps $f$ and $g$ are said to be (topologically) conjugate if there exists a homeomorphism $h: X \rightarrow Y$ such that $h \circ f=g \circ h$, where "o" denotes composition of two maps. For short, we call $f$ and $g h$-conjugate maps.

Some more results related to topological conjugation may be found in [10-12].

\section{Devaney Chaoticity}

Throughout this section, $f: X \rightarrow X$ and $g: Y \rightarrow Y$ are two $h$-conjugate maps defined on two topological spaces $X$ and $Y$, where $h: X \rightarrow Y$ is a homeomorphism.

Definition 3. The map $f$ is exact if, for every nonempty open set $U \subset X$, there exists some $m \in \mathbb{N}$ such that $f^{m}(U)=X$. The map $f$ is (topological) transitive (resp., mixing) if for any two nonempty open sets $U, V \subset X$, there exists some $m \in \mathbb{N}$ such that $f^{m}(U) \cap V \neq \phi$ (resp., $f^{n}(U) \cap V \neq \phi$ for all $n \geq m$ ). The map $f$ is weak mixing if $f \times f$ is transitive on $X \times X$.

Definition 4. (1) The map $f: X \rightarrow X$ is chaotic in the sense of Devaney if $f$ is transitive on $X$ and the set of periodic points of $f$ is dense in $X$.

(2) The map $f$ exhibits exact Devaney chaos (resp., mixing Devaney chaos and weakly mixing Devaney chaos) if $f$ is exact (resp., mixing and weakly mixing) and chaotic in the sense of Devaney. Devaney chaos, exact Devaney chaos, mixing Devaney chaos, and weak mixing Devaney chaos are briefly denoted by DevC, EDevC, MDevC, and WMDevC, respectively.

Similarly, from these definitions, we have these implications that (1) is right and its inverse is not proper on topological spaces.

Proposition 5. The map $f$ is exact if and only if $g$ is exact.

Proof. Necessity. Given any nonempty open set $V$ in $Y$, take $U=h^{-1}(V)$. Clearly, $U$ is a nonempty open set in $X$. Since $f$ is exact, there exists an $m \in \mathbb{N}$ such that $f^{m}(U)=X$. Noting that $f$ and $g$ are $h$-conjugate maps and that $h$ is a homeomorphism, it follows that

$$
g^{m}(V)=g^{m}(h(U))=h\left(f^{m}(U)\right)=h(X)=Y .
$$

This implies that $g$ is exact.

Similarly to the proof of necessity, the sufficiency follows immediately.

Proposition 6. The map $f$ is mixing if and only if $g$ is mixing.

Proof. Necessity. Given any two nonempty open sets $V_{1}, V_{2} \subset$ $Y$, it is clear that $U_{1}=h^{-1}\left(V_{1}\right)$ and $U_{2}=h^{-1}\left(V_{2}\right)$ are two nonempty open sets in $X$. Since $f$ is mixing, there exists $N \in$ $\mathbb{N}$ such that

$$
f^{n}\left(U_{1}\right) \cap U_{2} \neq \phi \quad \text { holds for all } n>N .
$$

This implies that

$$
\begin{aligned}
g^{n}\left(V_{1}\right) \cap V_{2} & =g^{n}\left(h\left(U_{1}\right)\right) \cap h\left(U_{2}\right) \\
& =h\left(f^{n}\left(U_{1}\right)\right) \cap h\left(U_{2}\right) \\
& \supset h\left(f^{n}\left(U_{1}\right) \cap U_{2}\right) \neq \phi .
\end{aligned}
$$

Thus, $g$ is mixing.

Sufficiency can be proved similarly.

Proposition 7. The map $f$ is weakly mixing if and only if $g$ is weakly mixing.

Proof. Necessity. For any two nonempty open sets $U, V \subset$ $Y \times Y$, according to the construction of product topology, it follows that there exist nonempty open sets $U_{1}, U_{2}, V_{1}, V_{2} \subset Y$ such that $U_{1} \times U_{2} \subset U$ and $V_{1} \times V_{2} \subset V$. Since $f$ is weakly mixing, there exists $n \in \mathbb{N}$ such that

$$
(f \times f)^{n}\left(h^{-1}\left(U_{1}\right) \times h^{-1}\left(U_{2}\right)\right) \cap\left(h^{-1}\left(V_{1}\right) \times h^{-1}\left(V_{2}\right)\right) \neq \phi .
$$

This implies that

$$
\begin{aligned}
(g \times g)^{n}(U) & \cap V \supset(g \times g)^{n}\left(U_{1} \times U_{2}\right) \cap\left(V_{1} \times V_{2}\right) \\
& =(g \times g)^{n}\left[(h \times h)\left(h^{-1}\left(U_{1}\right) \times h^{-1}\left(U_{2}\right)\right)\right] \\
& \cap\left[(h \times h)\left(h^{-1}\left(V_{1}\right) \times h^{-1}\left(V_{2}\right)\right)\right] \\
& =(h \times h)\left(f^{n}\left(h^{-1}\left(U_{1}\right)\right) \times f^{n}\left(h^{-1}\left(U_{2}\right)\right)\right) \\
& \cap\left[(h \times h)\left(h^{-1}\left(V_{1}\right) \times h^{-1}\left(V_{2}\right)\right)\right] \\
& \supset(h \times h)\left(\left[f^{n}\left(h^{-1}\left(U_{1}\right)\right) \times f^{n}\left(h^{-1}\left(U_{2}\right)\right)\right]\right. \\
& \left.\cap\left(h^{-1}\left(V_{1}\right) \times h^{-1}\left(V_{2}\right)\right)\right) \neq \phi .
\end{aligned}
$$

Thus, $g$ is weak mixing.

Sufficiency can be proved similarly.

Theorem 8 (see [3]). The map $f$ is DevC on $X$ if and only if $g$ is DevC on $Y$.

Proof. Necessity. Similarly to the proof of Proposition 6, it can be verified that $g$ is transitive on $Y$, as $f$ is transitive. According to the definition of periodic points, it is not difficult to check that

$$
\operatorname{Per}(g) \supset h(\operatorname{Per}(f))
$$

Applying this, one has

$$
\overline{\operatorname{Per}(g)} \supset \overline{h(\operatorname{Per}(f))} \supset h(\overline{\operatorname{Per}(f)})=h(X)=Y .
$$

This implies that $\overline{\operatorname{Per}(g)}=Y$.

Summing up the above discussion, it follows that $g$ is chaotic in the sense of Devaney.

Sufficiency can be proved similarly. 
Applying Propositions 5, 6, and 7 and Theorem 8, the following results are straightforward.

Theorem 9. The map $f$ is EDevC if and only if $g$ is EDevC.

Theorem 10. The map $f$ is MDevC if and only if $g$ is MDevC.

Theorem 11. The map $f$ is WMDevC if and only if $g$ is WMDevC.

Remark 12. According to the results obtained in this section, it is easy to see that DevC, EDevC, MDevC, and WMDevC are all preserved under topological conjugation on metric spaces. The next section will show that this does not hold for the LiYorke chaos.

\section{Li-Yorke Chaoticity}

First, we give the definition of Li-Yorke chaos.

Definition 13 (see [13]). Let $(X, d)$ be a metric space. A continuous map $f: X \rightarrow X$ is called to be chaotic in the sense of Li-Yorke if $P(f)=\mathbb{N}$, and there exists an uncountable set $S_{0} \subset X-\operatorname{Per}(f)$ such that

(i) $\lim \sup _{n \rightarrow \infty} d\left(f^{n}(x), f^{n}(y)\right)>0\left(\forall x, y \in S_{0}, x \neq y\right)$;

(ii) $\liminf _{n \rightarrow \infty} d\left(f^{n}(x), f^{n}(y)\right)=0\left(\forall x, y \in S_{0}\right)$;

(iii) $\lim \sup _{n \rightarrow \infty} d\left(f^{n}(x), f^{n}(p)\right)>0\left(\forall x \in S_{0}, \forall p \in\right.$ $\operatorname{Per}(f))$.

Next, we construct $f$-map and $g$-map and prove that they are topologically conjugative and that $f$-map is Li-Yorke chaotic, but $g$-map is not.

$f$-map. Let $X=[0,+\infty)$. Define $d_{1}: X \times X \rightarrow[0,1]$ as follows:

$$
d_{1}(x, y)= \begin{cases}0 & \text { if } x=y \\ \frac{1}{2^{[x]}} & \text { if }[x]=[y] \equiv 1(\bmod 2), x \neq y, \\ 1 & \text { others. }\end{cases}
$$

It is easy to see that $d_{1}$ is a metric on $X$. Define $f$ : $\left(X, d_{1}\right) \rightarrow\left(X, d_{1}\right)$ as follows: $f(0)=0, f(x)=x+1$ when $x \in X-\mathbb{Z}^{+}$; for every nature number $n \geq 2$,

$$
\begin{gathered}
f\left(\frac{n(n-1)}{2}+i\right)=\frac{n(n-1)}{2}+i+1 \quad \text { when } 0 \leq i \leq n-2, \\
f\left(\frac{n(n-1)}{2}+(n-1)\right)=\frac{n(n-1)}{2} .
\end{gathered}
$$

$g$-map. Let $X=[0,+\infty)$. Define $d_{2}: X \times X \rightarrow[0,1]$, where, for each pair $x, y \in X$,

$$
d_{2}(x, y)= \begin{cases}0, & \text { if } x=y \\ 1, & \text { if } x \neq y .\end{cases}
$$

Then $d_{2}$ is a metric on $X$. Define $g:\left(X, d_{2}\right) \rightarrow\left(X, d_{2}\right)$ such that $g(x)=f(x)$ for each $x \in X$.

Since both $d_{1}$ and $d_{2}$ are discrete metrics on $X, f$-map and $g$-map are both continuous.
Proposition 14. $f$-map and $g$-map are topologically conjugate.

Proof. Define $h:\left(X, d_{1}\right) \rightarrow\left(X, d_{2}\right)$ as $h(x)=x$ for any $x \in X$. It is easy to see that the map $h$ is a homeomorphism and that $h \circ f(x)=g \circ h(x)$ holds for any $x \in X$.

Proposition 15. $f$-map is Li-Yorke chaotic.

Proof. According to the definition of $f$-map, one has $f(0)=$ 0 , and again for any $n \geq 2$, there exists $x_{n}=n(n-1) / 2$ such that $f^{n}\left(x_{n}\right)=x_{n}$ and $f^{i}\left(x_{n}\right) \neq x_{n}$ for any $1 \leq i<n$. This implies that

$$
P(f)=\mathbb{N}, \quad \operatorname{Per}(f)=\mathbb{Z}^{+} .
$$

Take $S_{0}=(0,1) \cap(X-\operatorname{Per}(f))$. Now we assert that $S_{0}$ satisfies (i), (ii), and (iii) of Definition 13.

(i) For any $x, y \in S_{0}$ with $x \neq y$, we have

$$
\begin{aligned}
1 & >\limsup _{n \rightarrow \infty} d_{1}\left(f^{n}(x), f^{n}(y)\right) \\
& =\limsup _{n \rightarrow \infty} d_{1}(x+n, y+n) \\
& \geq \limsup _{n \rightarrow \infty} d_{1}\left(f^{2 n}(x), f^{2 n}(y)\right) \\
& =\lim _{n \rightarrow \infty} d_{1}(x+2 n, y+2 n)=1 .
\end{aligned}
$$

That is, $\lim _{\sup } \operatorname{si\infty }_{n} d_{1}\left(f^{n}(x), f^{n}(y)\right)=1>0$.

(ii) Consider

$$
\begin{aligned}
& \liminf _{n \rightarrow \infty} d_{1}\left(f^{n}(x), f^{n}(y)\right) \\
& \quad=\liminf _{n \rightarrow \infty} d_{1}(x+n, y+n) \\
& \quad \leq \lim _{n \rightarrow \infty} d_{1}(x+2 n+1, y+2 n+1) \\
& \quad \leq \lim _{n \rightarrow \infty} \frac{1}{2^{2 n+1}}=0 .
\end{aligned}
$$

(iii) For any $x \in S_{0}$ and any $p \in \operatorname{Per}(f)=\mathbb{Z}^{+}$, it is easy to see that there exists a $N \in \mathbb{N}$ such that $f^{n}(p) \leq N$ and that $f^{n}(x)=x+n \geq N+1$ for any $n>N+1$. Then, for any $n \geq N+1, d_{1}\left(f^{n}(x), f^{n}(p)\right)=1$.

So,

$$
\limsup _{n \rightarrow \infty} d_{1}\left(f^{n}(x), f^{n}(p)\right)=1>0 .
$$

We thus conclude that $f$-map is chaotic in the sense of Li-Yorke.

Proposition 16. g-map is not Li-Yorke chaotic.

Proof. For any $x, y \in X$ with $x \neq y$, it is clear that for any $n \in \mathbb{N}, d_{2}\left(g^{n}(x), g^{n}(y)\right)=1$. Then,

$$
\liminf _{n \rightarrow \infty} d_{2}\left(g^{n}(x), g^{n}(y)\right)=1 \text {. }
$$

This implies that $g$-map is not Li-Yorke chaotic. 
The following theorem holds obviously by Propositions 14,15 , and 16.

Theorem 17. For a continuous self-map which is defined on a metric space, Li-Yorke's chaoticity is not preserved under topological conjugation.

Remark 18. Section 2 discusses some notions of "chaos" which can be defined on topological spaces and Section 3 studies Li-Yorke chaos on metric spaces. There are some other problems for further research; For example, could LiYorke chaos be extended to topological spaces? According to Section 3, we can conclude that even if Li-Yorke chaos may be extended to topological spaces, topological conjugation does not preserve it.

\section{Acknowledgments}

This work was financially supported by the Scientific Research Fund of Sichuan Provincial Education Department (12ZA098) and Artificial Intelligence of Key Laboratory of Sichuan Province (2012RYY04).

\section{References}

[1] T. Y. Li and J. A. Yorke, "Period three implies chaos," The American Mathematical Monthly, vol. 82, no. 10, pp. 985-992, 1975.

[2] R. L. Devaney, An Introduction to Chaotic Dynamical, Addison Wesley, New York, NY, USA, 1989.

[3] J. Banks, J. Brooks, G. Cairns, G. Davis, and P. Stacey, "On Devaney's definition of chaos," The American Mathematical Monthly, vol. 99, no. 4, pp. 332-334, 1992.

[4] E. Akin, Recurrence in Topological Dynamics: Furstenberg Families and Ellis Actions, Plenum Press, New York, NY, USA, 1997.

[5] H. Furstenberg, Recurrence in Ergodic Theory and Combinatorial Number Theory, Princeton University Press, Princeton, NJ, USA, 1981.

[6] D. Kwietniak and M. Misiurewicz, "Exact Devaney chaos and entropy," Qualitative Theory of Dynamical Systems, vol. 6, no. 1, pp. 169-179, 2005.

[7] P. Oprocha, "Relations between distributional and Devaney chaos," Chaos, vol. 16, no. 3, Article ID 033112, 2006.

[8] A. Crannell, "A chaotic, non-mixing subshift," Discrete and Continuous Dynamical Systems, vol. 43, pp. 195-202, 1998.

[9] J. Banks, "Regular periodic decompositions for topologically transitive maps," Ergodic Theory and Dynamical Systems, vol. 17, no. 3, pp. 505-529, 1997.

[10] A. Bielecki, “Topological conjugacy of discrete time-map and Euler discrete dynamical systems generated by a gradient flow on a two-dimensional compact manifold," Nonlinear Analysis: Theory, Methods \& Applications, vol. 51, no. 8, pp. 1293-1317, 2002.

[11] N. H. Kuiper, "Topological conjugacy of real projective transformations," Topology, vol. 15, no. 1, pp. 13-22, 1976.

[12] S. M. Shea, "Topological conjugacy on the complement of the periodic points," Indagationes Mathematicae, vol. 23, no. 3, pp. 300-310, 2012.

[13] C. Tian and G. Chen, "Chaos in the sense of Li-Yorke in coupled map lattices," Physica A, vol. 376, pp. 246-252, 2007. 


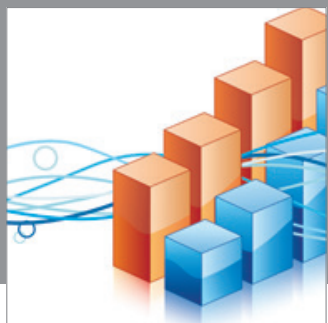

Advances in

Operations Research

mansans

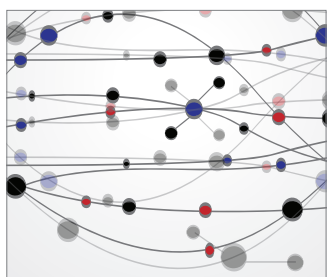

The Scientific World Journal
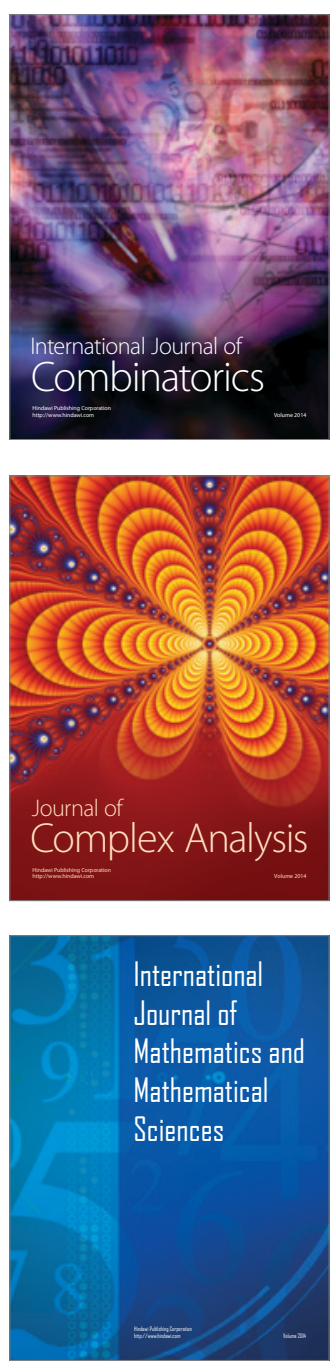
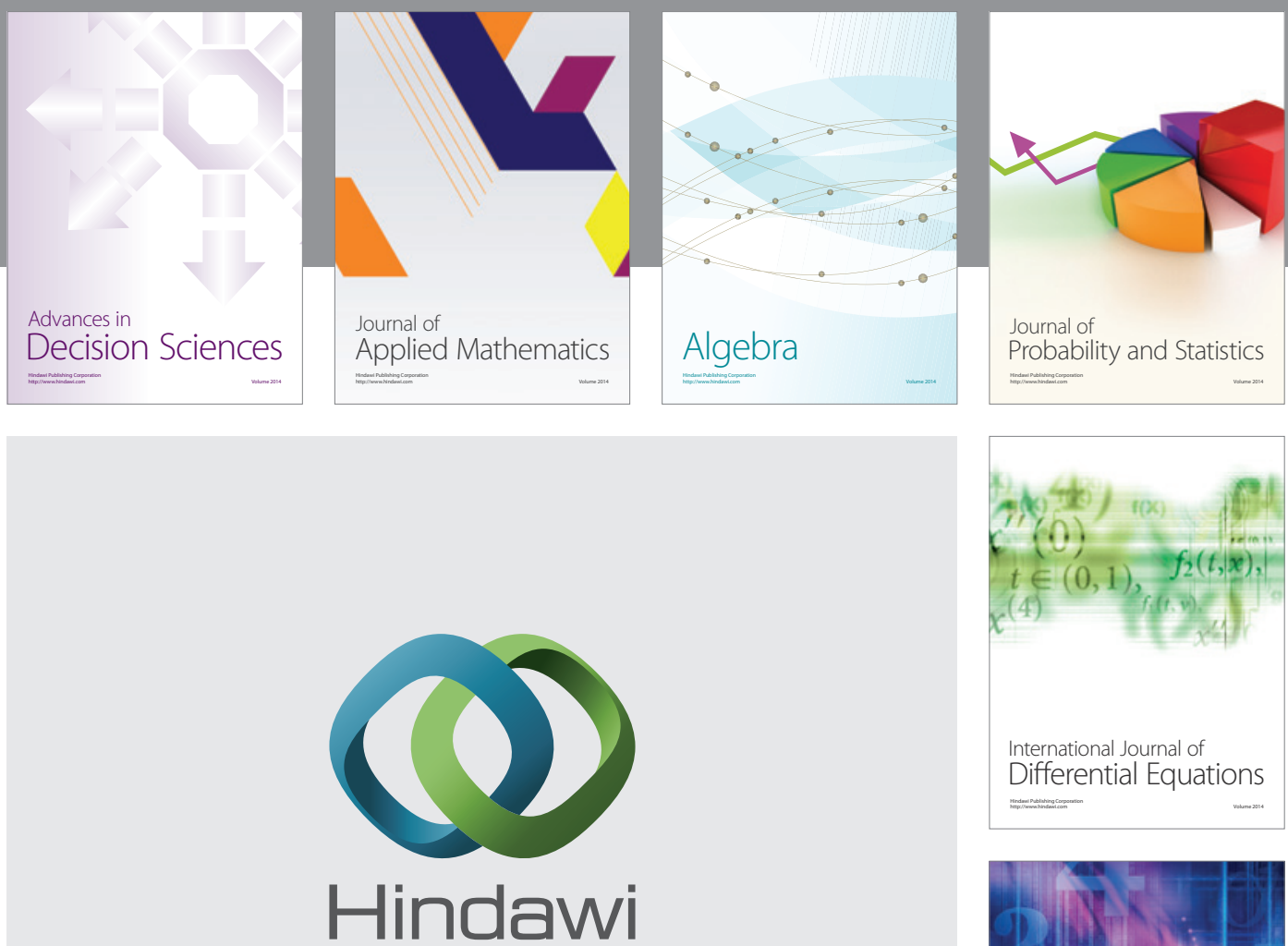

Submit your manuscripts at http://www.hindawi.com
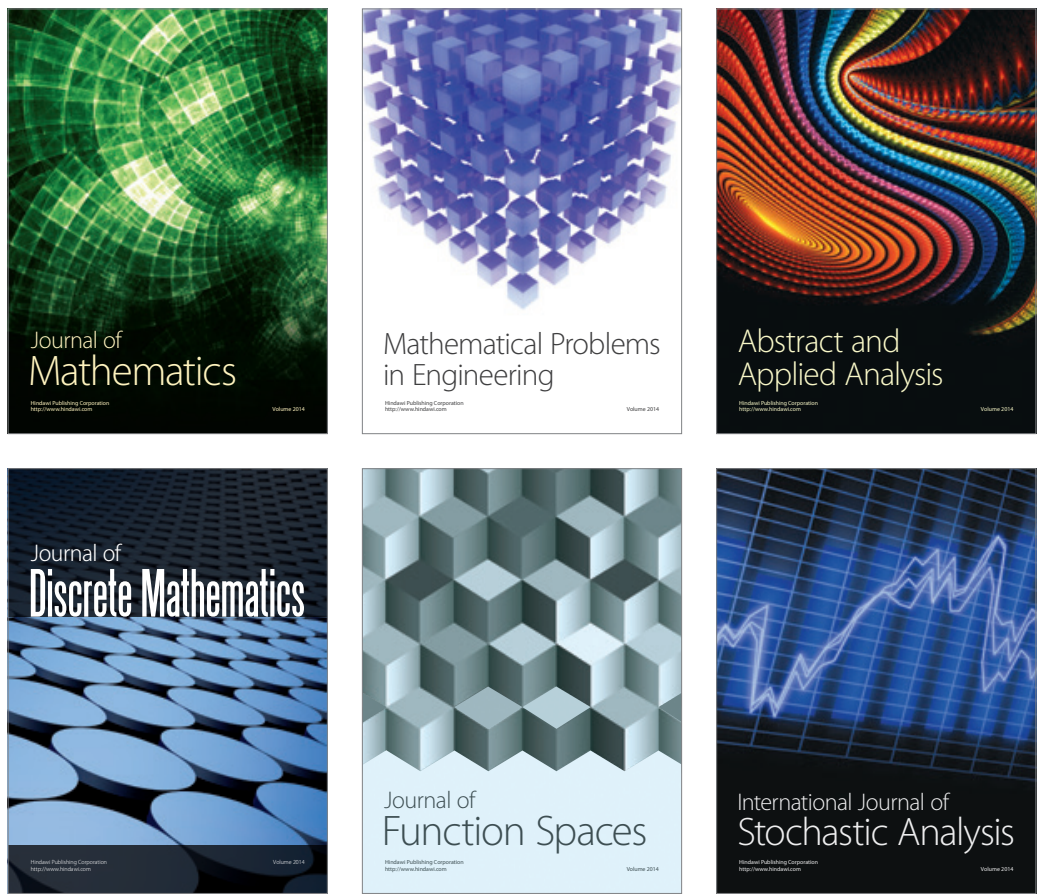

Journal of

Function Spaces

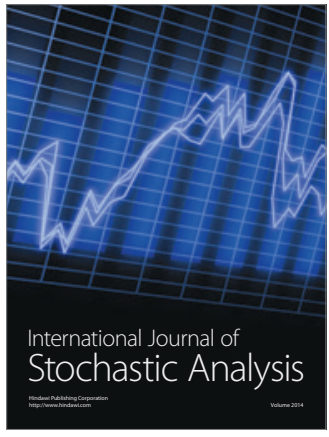

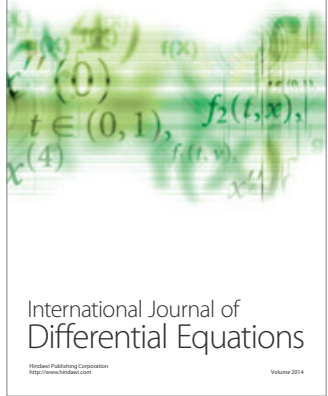
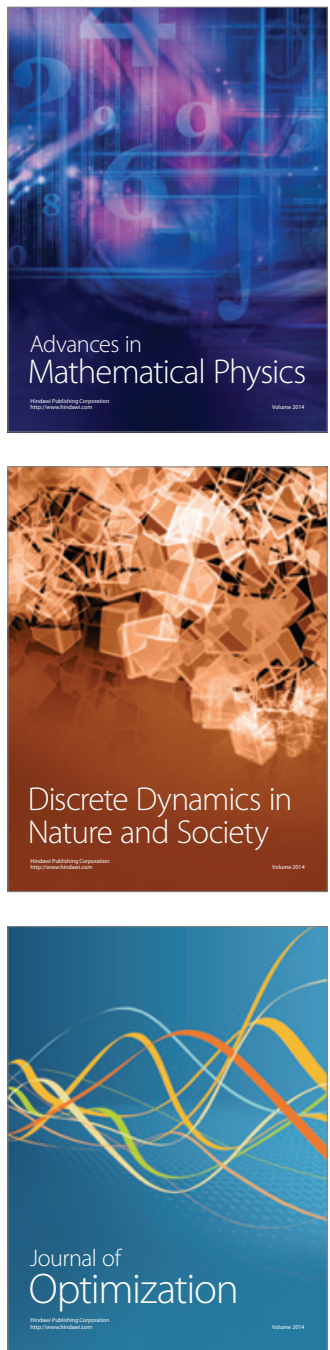